\title{
Application of an indocyanine green surgical fluorescence imaging system in sentinel lymph node biopsy of acral malignant melanoma
}

\author{
Keyu Jiang ${ }^{1 \#}$, Binlin Luo ${ }^{1 \#}$, Zuoqiong Hou ${ }^{1}$, Chujun Li $^{1}$, Huiming Cai ${ }^{2}$, Jian Tang ${ }^{1}$, Gang Yao ${ }^{1}$ \\ ${ }^{1}$ Department of Plastic and Burn Surgery, The First Affiliated Hospital with Nanjing Medical University, Nanjing, China; ${ }^{2}$ Nanjing Nuoyuan \\ Medical Devices Co., Ltd., Nanjing, China \\ Contributions: (I) Conception and design: J Tang, G Yao; (II) Administrative support: K Jiang, B Luo; (III) Provision of study materials or patients: \\ B Luo, Z Hou; (IV) Collection and assembly of data: K Jiang, C Li, H Cai; (V) Data analysis and interpretation: K Jiang, J Tang; (VI) Manuscript \\ writing: All authors; (VII) Final approval of manuscript: All authors. \\ "These authors contributed equally to this work. \\ Correspondence to: Jian Tang; Gang Yao. Department of Plastic and Burns Surgery, The First Affiliated Hospital of Nanjing Medical University, 300 \\ Guangzhou Rd, Nanjing 210029, Nanjing, China. Email: near_spine@163.com; yaogang2005@hotmail.com.
}

\begin{abstract}
Background: Regional lymph node status is an independent influencing factor for the prognosis of acral malignant melanoma, and the accuracy of sentinel lymph node biopsy (SLNB) is directly related to the judgment of regional lymph node status. This study aimed to explore the application value of indocyanine green (ICG) surgical fluorescence imaging system in the SLNB of acral malignant melanoma.

Methods: A total of 34 patients with acral malignant melanoma were admitted to the Department of Burn and Plastic Surgery in Jiangsu Provincial People's Hospital from January 2020 to March 2020. Among these patients, 22 required SLNB. ICG and methylene blue $(\mathrm{MB})$ were combined to intraoperatively trace the sentinel lymph nodes (SLNs). The total number of SLNs detected during the operation was counted. We compared the number, detection rate, as well as the detection rate and false negative rate of positive SLNs of SLNs detected by ICG, MB, and ICG combined with MB.

Results: A total of 56 SLNs were detected in the 22 patients, among which 55 were detected by ICG (98\%), 41 were detected by MB (71\%), and 56 (100\%) were detected by ICG combined with MB, and the average number of SLNs were 2.5, 1.64, and 2.55, respectively. A total of nine SLNs were detected, of which nine were detected by ICG (100\%), seven by MB (78\%), and nine by ICG combined with MB (100\%). Patients with negative SLNs had no recurrence at the 6-month follow-up.

Conclusions: Compared with MB, the ICG fluorescent imaging system can improve the detection rate of SLNs in patients with acral malignant melanoma. Also, ICG combined with MB was superior to ICG alone.
\end{abstract}

Keywords: Acral malignant melanoma; sentinel lymph node biopsy (SLNB); indocyanine green (ICG); methylene blue (MB)

Submitted Jul 23, 2021. Accepted for publication Sep 18, 2021.

doi: 10.21037/atm-21-4366

View this article at: https://dx.doi.org/10.21037/atm-21-4366

\section{Introduction}

Globally, the incidence of melanoma is increasing every year. In 2020, the most recent statistics from the United States reported 100,350 new cases, with 6,850 deaths, and the number of new cases in China has reached
20,000 annually (1). Sentinel lymph nodes (SLNs) are the first echelon lymph nodes on the lymphatic metastasis pathway of melanoma, and their status is an important prognostic factor for malignant melanoma (2). SLN biopsy (SLNB) was introduced by Morton et al. in 1992. With 
SLNB, we can not only identify the regional lymph node status, but also avoid the postoperative complications caused by prophylactic lymphadenectomy (3). In 2001, SLN status was included in the melanoma staging system by the American Cancer Society, and SLNB is also considered to be the standard treatment for patients with malignant melanoma (4). The Multicenter Selective Lymphadenectomy Trials indicate that there are no overall or melanoma-specific survival advantages to performing SLNB followed by immediate completion lymph node dissection compared with wide excision and observation for patients with positive sentinel nodes. SLNB should be considered as a staging procedure which can help patients to be stratified and entered into adjuvant trials and trials of new drugs for the prevention of melanoma progression (5).

Commonly used SLNB tracers include blue dyes such as lymphazurin, patent blue, methylene blue (MB), and ${ }^{99} \mathrm{~m}$ Tc radioactive colloid, etc. (6-8). At present, the dual tracer scheme of a radioisotope combined with a blue dye is the gold standard technology (9). However, there are some problems in the application of radioactive isotopes, including the high cost, complex waste disposal, tedious staff training, and radiation exposure risk for medical staff and patients (10). Also, the application of blue dye involves problems such as low SLN detection rate, high false negative rate, as well as the risk of allergy, skin staining, ulcer, and other adverse reactions $(11,12)$. Therefore, a new tracer method for SLN localization is urgently needed.

Indocyanine green (ICG), as a cyanine fluorescent molecule, emits near-infrared wavelengths of fluorescence after excitation by light of a specific wavelength, which can be detected by near-infrared imaging equipment. ICG has been preliminarily used for the localization of SLN in breast, uterine, and gastric cancers, etc., and has shown important advantages and potential (13-15). Our research is aimed at patients with acral melanoma. In previous studies, acral melanoma patients were rarely distinguished from patients with skin melanoma. In previous studies, ICG cannot replace radionuclides in SLNB tracking, but the difference is mainly concentrated in the complicated lymphatic drainage areas such as the head, face and trunk (16). In the limbs with relatively simple lymphatic drainage, if ICG can maintain a high detection rate and a low false negative rate, then for patients with acral melanoma in areas with underdeveloped economies and poor medical conditions, ICG or ICG combined with blue dyes maybe the best choice for SLNB, and can even promote the innovation of medical guidelines. The main objective of this observational study was to investigate the tracer efficacy of ICG and MB in SLNB in patients with acral malignant melanoma. The patients were analyzed by self-control, and the number of SLNs detected, detection rate, positive lymph node detection rate, and false negative rate of the two tracers in SLNB were respectively observed. We present the following article in accordance with the STROBE reporting checklist (available at https:// dx.doi.org/10.21037/atm-21-4366).

\section{Methods}

The study was conducted in accordance with the Declaration of Helsinki (as revised in 2013). All the procedures were approved by local Ethics Committee of First Affiliated Hospital with Nanjing Medical University (2019-447). All participants signed the informed consent.

\section{Study design, setting, and patients}

This study was designed as a clinical prospective cohort study. A total of 34 patients with acral malignant melanoma were enrolled in the Department of Plastic and Burn Surgery of Jiangsu Provincial People's Hospital from January 2020 to March 2020. Among these, 22 patients meeting the criteria were eventually included. The inclusion criteria were as follows: (I) patients with pathologically-diagnosed acral malignant melanoma; (II) patients with no clinical or radiographic evidence of lymph node metastasis; (III) patients aged $>18$ years old; (IV) $\mathrm{T}$ stage $\geq \mathrm{Ib}$; and (V) $\mathrm{T}$ stage $<\mathrm{Ib}$, but with ulcer (17). Patients were exclusion based on the following criteria: (I) those with a previous surgical history in the SLN area; and (II) those with ICG contraindications, such as pregnancy, lactation, and thyroid diseases. The patients were diagnosed as malignant melanoma by an independent review of radiographs by two pathologists, and no SLN enlargement was determined by physical examination by two senior clinicians. Preoperative imaging confirmed that there were no signs of regional lymphatic metastasis or systemic metastasis. The patients' demographic characteristics, clinical diagnosis, $\mathrm{T}$ stage of primary tumor, Breslow thickness of tumor, presence of ulcer, and other information were collected. The total number of SLNs detected and the numbers of SLNs detected by the two tracer methods were recorded intraoperatively.

\section{Fluorescence imaging system for ICG surgery and MB}

The ICG Surgical Fluorescent Imaging System was 

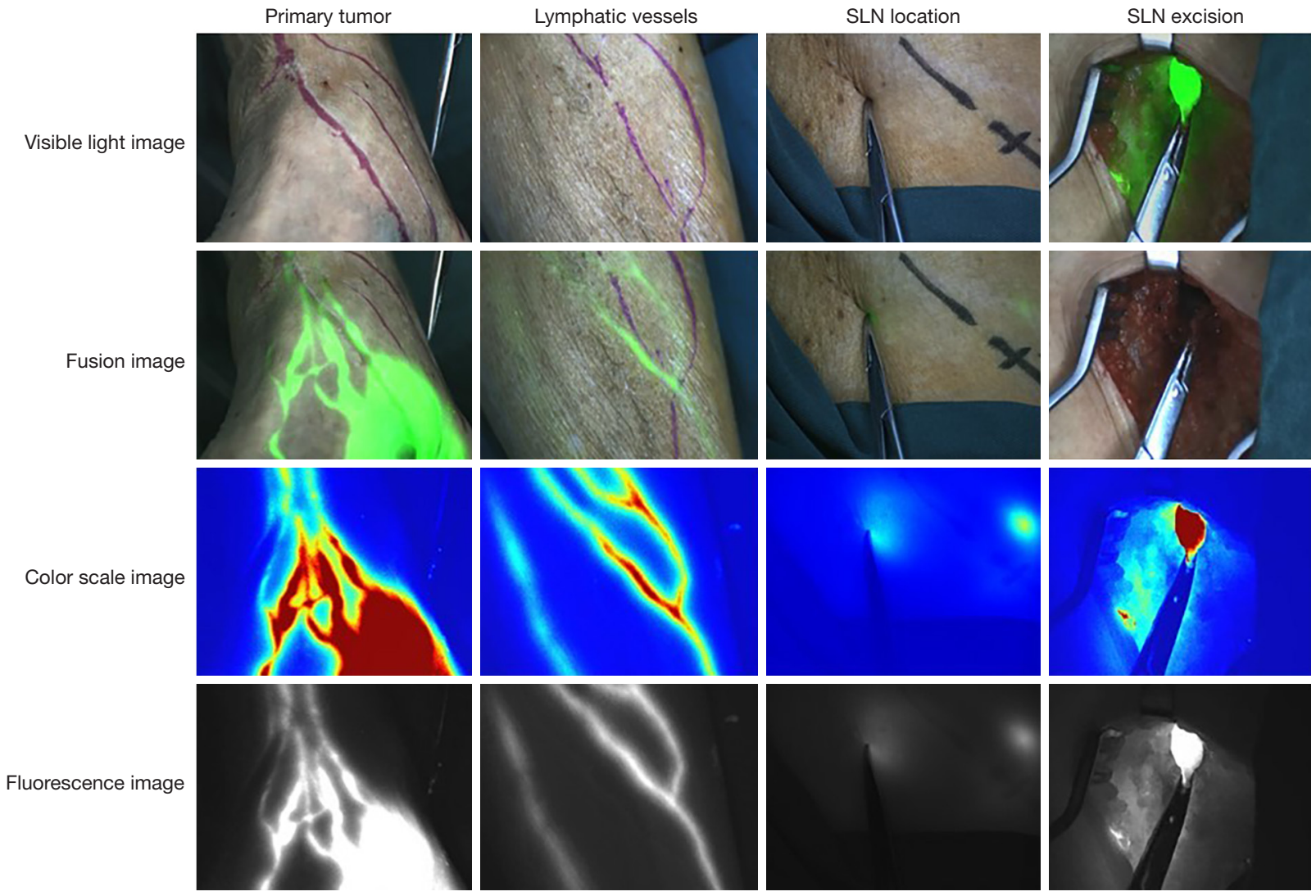

Figure 1 Intraoperative ICG fluorescence imaging of a patient with lower extremity malignant melanoma. ICG, indocyanine green; SLN, sentinel lymph node.

provided by Nanjing Nuoyuan Medical Equipment Co., Ltd. (Nanjing, China). Specifically, the Surgical Fluorescent Imaging System REAL-IGS FLA-10 series was used, which consists of two types of light sources: a "white" LED that illuminates the surgical area, and a near-infrared LED that emits $760 \mathrm{~mm}$ fluorescent excitation light. Using the multi-mode optical path system, three kinds of optical signals (fluorescence, laser, and visible light) were simultaneously collected. After being processed by the customized intelligent system, the three kinds of optical signals were respectively displayed as a fluorescence image, color gradation image, and visible light image in real time. These three images could either be displayed individually or superimposed. MB was provided by Suzhou Jizhou Pharmaceutical Co., Ltd. (Suzhou, China).

\section{Surgery}

Endotracheal intubation general anesthesia or local infiltration anesthesia were used according to the patient's age, basic condition, and site of the primary lesion. SLNB was performed in all cases where the primary lesion and regional lymph node sites were fully exposed intraoperatively.

As shown in Figures 1,2, intradermal injection was performed in four quadrants around the primary tumor, and $1 \mathrm{~mL} \mathrm{MB}$ dye and $1 \mathrm{~mL}(2.5 \mathrm{mg} / \mathrm{mL})$ ICG were respectively injected at the same injection site. After local massage for $5 \mathrm{~min}$, the ICG was stimulated by the surgical fluorescence imaging system and the fluorescence was detected. The SLN position was determined by real-time fluorescence indicating the lymphatic drainage route, and the skin incision was designed. If no fluorescent lymph nodes were detected, conventional groin and axillary incisions were designed for exploration.

SLNs were successfully found when the following conditions were observed: (I) lymphatic flow from the primary tumor to the lymph nodes as indicated by 

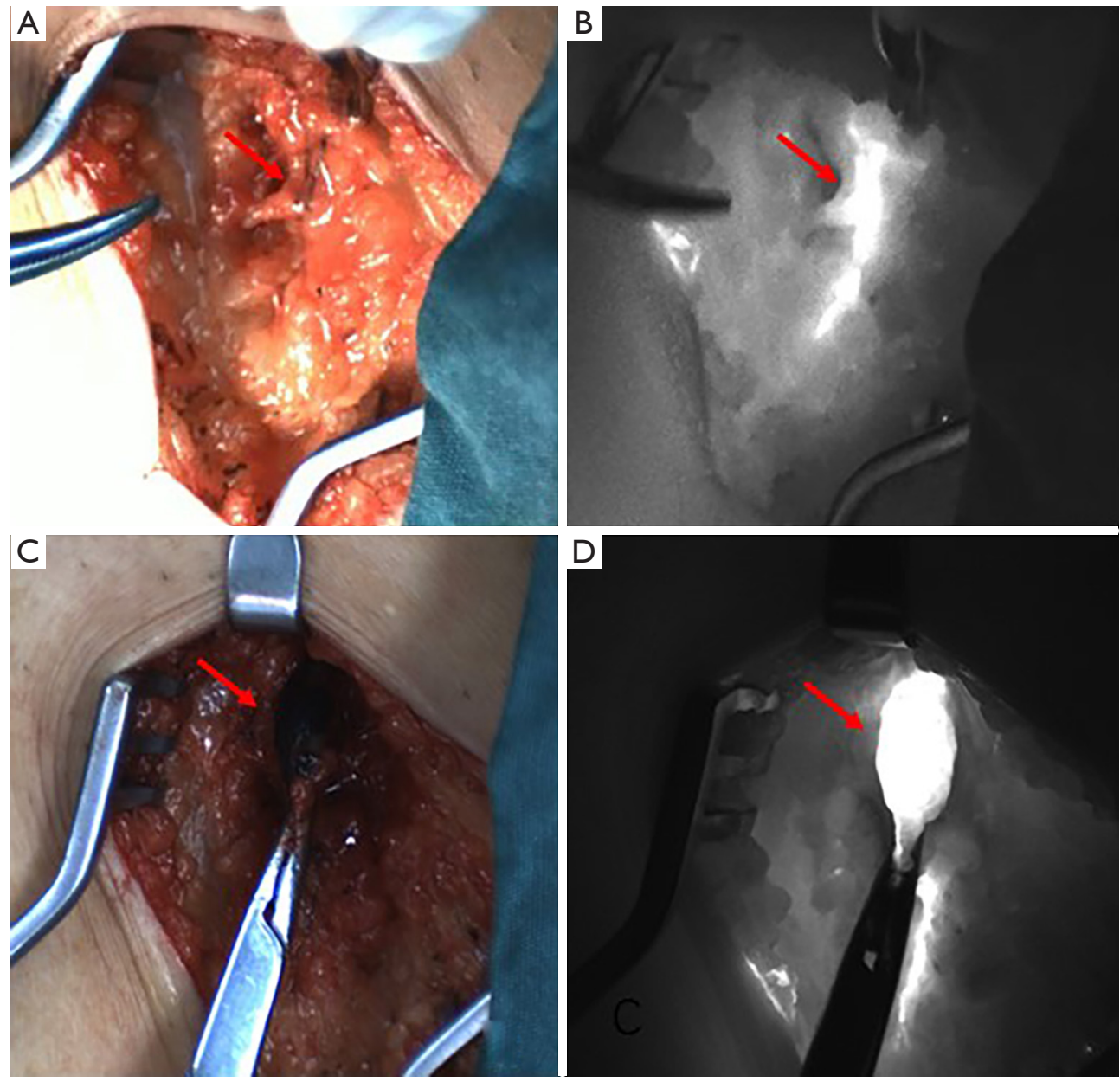

Figure 2 Representative SLN detecting images of ICG, MB. The red arrow marks the lymphatic vessels and lymph nodes that are traced. ICG, indocyanine green; MB, methylene blue; SLN, sentinel lymph node.

fluorescence; and (II) lymph nodes stained blue by MB. After resection of the fluorescent and/or blue-stained lymph nodes, the surgical area was examined for residual fluorescent or blue nodules. The near infrared fluorescence and blue staining of the resected SLNs were observed. Routine analysis was performed by histopathology. Drainage strips were placed at the surgical site and bandaged under pressure. Postoperative local skin lesions, nodule formation, and allergic reactions were considered adverse reactions to surgery.

\section{Statistical analysis}

Normally distributed data were presented as the mean \pm $\mathrm{SD}$, and the non-normally distributed data were presented as the median. Statistical analysis was performed using
GraphPad 8.0 (GraphPad Software, La Jolla, CA, USA). Differences in the number of SLNs identified by ICG and $\mathrm{MB}$ were analyzed, and $\mathrm{P}<0.05$ was considered statistically significant.

\section{Results}

As shown in Table 1, the ages of patients at diagnosis ranged from 31 to 86 years (median: 56.5 years). There were 12 cases in males and 10 cases in females [body mass index (BMI) 23.53 3.89 ]. The primary tumor sites included the following: hand, one case; finger, four cases; foot, 12 cases; and toe, five cases. The Breslow thickness was $4.94 \pm 3.67 \mathrm{~mm}$, and $64 \%$ of the patients had ulcerative melanomas. SLN location could be determined by ICG imaging prior to epidermal incision in $91 \%$ of patients. 
Table 1 Tumor characteristics of 22 patients with melanoma

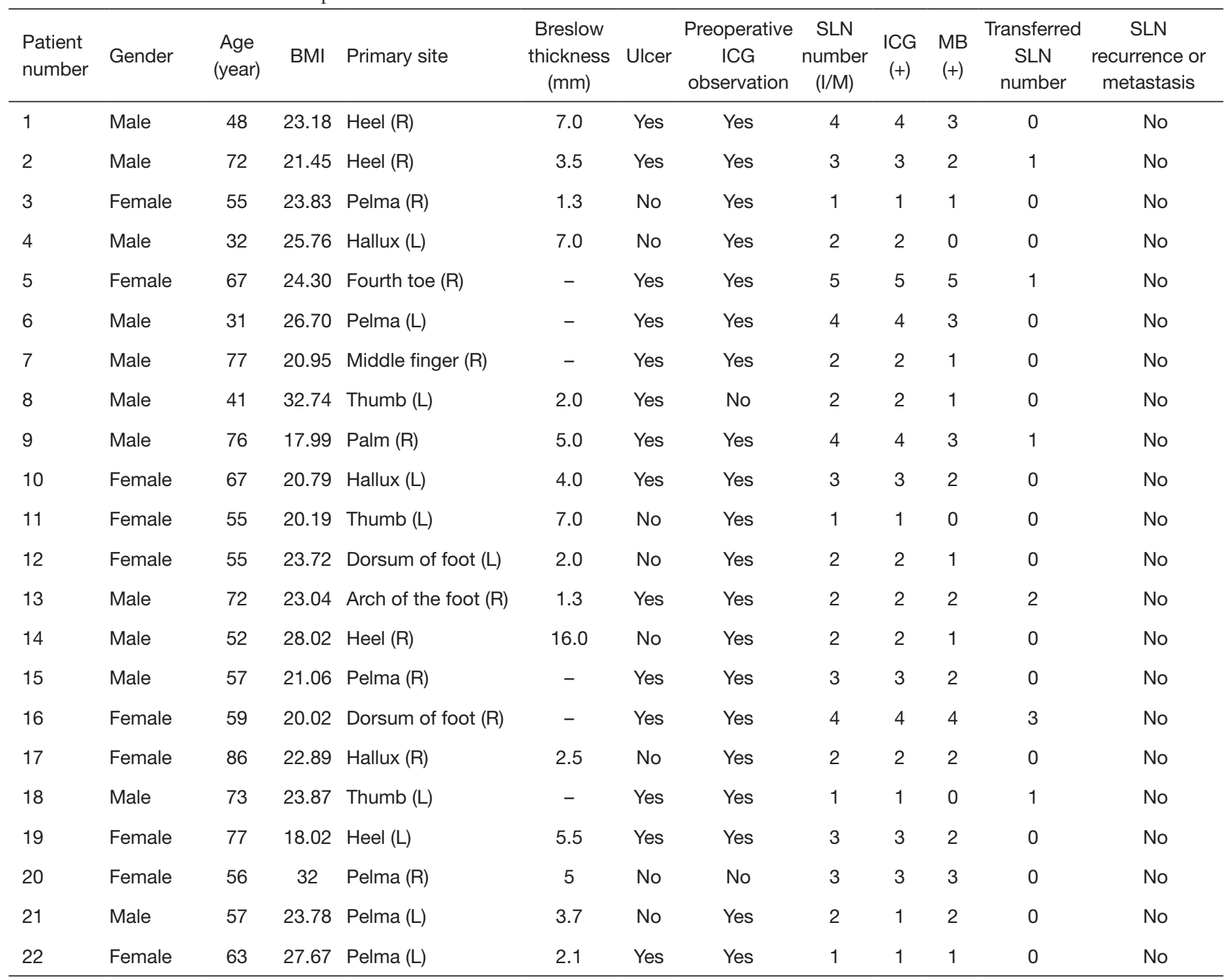

ICG, indocyanine green; SLN, sentinel lymph node; BMI, body mass index; MB, methylene blue; R, right; L, left.

\section{Treatment differences between the study groups}

The SLN detection rates of different tracers are shown in Table 2. A total of 56 SLNs were detected in the 22 patients, of which 55 SLNs were detected in 22 patients by ICG (98\%), 41 SLNs were detected in 19 patients by MB (73\%), and 56 SLNs were detected in 22 patients by ICG and MB (100\%). The SLN detection rate was significantly different between ICG and MB $(\mathrm{P}<0.0001)$. Four SLNs in three patients $(7 \%)$ were identified by ICG only, while one SLN in one patient (2\%) was identified by MB only. The average number of SLNs detected per patient was 2.8 in ICG, 1.8 in MB, and 2.8 in ICG + MB. The average number of SLNs detected by ICG was significantly higher than that by MB. The ICG identified 9 (100\%) metastatic SLNs in all six patients, the MB identified 8 (89\%) metastatic SLN in five patients, and the ICG + MB identified 9 (100\%) metastatic SLN in all six patients. It could be seen that the combination of ICG + MB had a higher SLN and metastatic lymph node detection rate than ICG or MB alone. All the patients were followed up for at least 6 months. No false negative SLN was observed, and no adverse reactions were observed due to the tracer.

\section{Discussion}

Malignant melanoma patients in China are mainly acrotype 
Table 2 Detection rate of lymph nodes and metastatic lymph nodes with different tracers

\begin{tabular}{|c|c|c|}
\hline & Patient number & SLN number \\
\hline $\operatorname{ICG}(+)$ & 22 & 55 \\
\hline $\mathrm{MB}(+)$ & 19 & 41 \\
\hline $\mathrm{ICG}(+) \mathrm{MB}(-)$ & 3 & 4 \\
\hline \multicolumn{3}{|l|}{ Detection rate } \\
\hline ICG & $22(100 \%)$ & 55 (98\%) \\
\hline MB & $19(86 \%)$ & 41 (73\%) \\
\hline$I C G+M B$ & $22(100 \%)$ & $56(100 \%)$ \\
\hline MB & $5(23 \%)$ & 8 (89\%) \\
\hline$I C G+M B$ & $6(27 \%)$ & $9(100 \%)$ \\
\hline
\end{tabular}

ICG, indocyanine green; SLN, sentinel lymph node; MB, methylene blue.

and mucosal type, which are often characterized by deep lesion invasion, high incidence of ulcers, high rate of lymph node metastasis, poor prognosis, etc. Therefore, understanding the status of SLNs is crucial for determining the stage and prognosis of melanoma patients (18).

The cutaneous lymphatic drainage pathway is very complex. Leong et al. analyzed the SLN drainage pathway in 400 patients with malignant melanoma using radionuclide imaging. They classified the SLN drainage pathway pattern as I-VII, where lymphatic vessels converge or branch and eventually merge into one or more lymph nodes. In patients with acral melanoma, although the position of regional lymph nodes is relatively fixed, multiple SLNs can still be developed at the same time (19). This greatly increases the surgical difficulty and false negative rate of SLNB. If a patient with SLN metastasis is misdiagnosed, they often do not receive more rigorous follow-up before clinical or radiographic recurrence, and do not receive timely followup treatment. Although SLNB is performed by skilled surgeons using standard biopsy procedures and SLN recognition rates can reach $95 \%$ or more, approximately $5 \%$ of SLN-negative patients develop local or systemic metastases $(20,21)$.

The following four factors effected the detection rate, the first is the characteristics of the patient and the tumor, such as the patient's tumor location, tumor burden, degree of obesity, etc. Usually, the lymphatic drainage of the head, face, neck, and trunk is quite complicated, and it is often impossible to find every lymphatic drainage basin with simple $\mathrm{MB}$ tracing. If the patient's primary tumor has a high tumor burden, a large number of tumor cells will metastasize and fill up the sentinel lymph nodes, making the macromolecular tracer unable to trace accurately, and obese patients have too much subcutaneous fat, which affects the visualization of ICG traces and affects the detection rate. The second is the type, concentration and dose of the tracer. Each tracer method has its optimal concentration and dose, and affects the detection of SLN. The third is the operating accuracy and proficiency of the surgeon. After the surgeon can perform SLNB operations proficiently, the SLN detection rate has been significantly improved $(16,22)$.

The factors leading to false negative SLN are generally divided into two types: the accuracy of SLN detection and the sensitivity of tumor cells in the resected SLN detection. Melanoma cells can deposit in lymph nodes and block lymphatic vessels, impede SLN recognition, or the tumor cells may move slowly and do not reach SLN during SLNB $(20,21,23,24)$. Even if the SLN is removed accurately, inaccurate pathological results often lead to false negative SLNs, which may be caused by a small tumor load in lymph nodes or the poor sensitivity of standard hematoxylin and eosin staining $(25,26)$. Therefore, the detection level of 
SLN metastases can be improved by immunohistochemical staining for S100, HMB-45, and MART-1 (27).

ICG is a small molecule of tricarbocyanine dye that is often used in the diagnosis and treatment of patients with ophthalmological, cardiology and liver diseases (28). To date, use of NIR ICG-guided SLN biopsy has been performed in breast, skin, gastric, colorectal, anal, and lung cancers. In 2009, Fujiwara et al. first applied ICG in SLNs of skin cancer, and achieved excellent mapping performance (29). ICG has a low allergic risk, is relatively easy to operate and administer, does not expose patients to radiation, and significantly reduces allergic reactions compared to reactive dyes $(0.05 \%$ and $1.1 \%$, respectively) $(30,31)$. Due to its low molecular weight, ICG can usually reach the lymph drainage area within $15 \mathrm{~min}$ (16). With the help of the nearinfrared imaging system, ICG can penetrate the soft tissue $(1-2 \mathrm{~cm})$, and conduct real-time observation of lymphatic vessels and lymph nodes. SLN of fluorescence development could be observed in $91 \%$ of patients without incision of the epidermis. Real-time development has enabled more accurate surgical positioning and more detailed anatomy.

Compared with the MB tracer method, blue-stained lymphatic vessels need to be located first, and the SLN can be anatomized anterograde according to the lymphatic vessels. The precise positioning of ICG reduces surgical injury and the incidence of lymphedema at the surgical site, and improves the surgical satisfaction of patients (31). Although ICG has a short half-life, it can persist in lymph nodes for more than 10 hours, which provides a clearer boundary for subsequent regional lymph node dissection (16). To sum up, it is clear that ICG offers unique advantages over reactive dyes.

In our study, ICG was added on the basis of the widely used MB tracer method to explore whether this method could improve the SLN recognition performance in patients with early acral malignant melanoma. We found that compared with MB, ICG significantly increased the SLN detection rate (73\% vs. $98 \%$, respectively), which was also consistent with previous studies (32). Due to the small size of ICG molecules, ICG can be better localized in SLNs filled with tumor cells (22). The high recognition rate of ICG offers the possibility of a non-radioactive alternative to SLN tracers.

In addition to the superior SLN detection rate, ICG also plays an important role in reducing false negative lymph nodes. In our patients, the ICG identified metastatic SLNs that were not recognized by $\mathrm{MB}$, and no SLN-negative patients had local lymph node recurrence at present, possibly due to the high sensitivity of the ICG, which detected more SLNs. In this study, the average number of SLNs detected by ICG combined with MB was 2.8, which was higher than the average number of SLNs detected by $\mathrm{MB}$ alone, and is consistent with previous studies $(33,34)$. We speculated that the visibility of dyes by high-resolution infrared devices was much higher than direct visual observation, and thus, ICG identified the SLNs missed by $\mathrm{MB}$ and improved the accuracy of the biopsy procedure.

In addition, we found that ICG can also detect in-transit metastasis of tumors, which are cutaneous, subcutaneous, or soft tissue metastatic nodules that metastasize through lymphatic vessels between the primary lesion (beyond $2 \mathrm{~cm}$ in diameter) and regional lymph nodes $(35,36)$. During SLNB, one patient accidentally found the ICG fluorescence concentration point in the forearm, which was pathologically diagnosed as transitional metastasis after surgical resection. In the two SLNs taken from another patient, ICG identified only one of them, while MB identified both SLNs, and therefore, ICG could not completely replace the tracer role of MB in SLNB. We speculated that the missed SLN detection might have been caused by visual error caused by the overlap of lymph nodes.

All in all, compared to other staining technology, ICG has obvious advantages. First: Compared with MB, ICG significantly improves the detection rate of SLN, and its detection rate is similar to that of radioactive colloid in many studies. Second: Compared with MB, the incidence of severe allergic reactions caused by ICG is only $0.05 \%$, and compared with radioactive colloid, it eliminates the potential risk of radiation exposure. Third: Due to the intraoperative visualization characteristics of ICG, it can accurately locate the SLN, shorten the operation time, reduce surgical damage, and reduce the risk of postoperative complications (16,30,31).

In this study, the ICG near infrared imaging system was firstly applied in China to perform SLNB on patients with acral malignant melanoma, confirming the effectiveness and safety of ICG. This study is of highly significant for the promotion of ICG in China. However, there were still shortcomings in this study that should be noted. Firstly, due to limited conditions, we did not apply standard radionuclide imaging methods in our study, so we could not directly compare the detection efficiency of ICG and standard tracer schemes. Secondly, the median followup time in this study was less than 1 year, so the incidence of SLN false negatives could not be adequately observed. Since this was a self-controlled trial, the surgeon may 
have been inclined to end the operation after detecting all of the fluorescent lymph nodes, rather than continue to look for lymph nodes that may be blue stained, which will underestimate the detection efficiency of $\mathrm{MB}$. We have to admit that the number of cases in the study does not make our results look very accurate, but most Chinese patients with acral melanoma have heavy tumor burden, and many patients have clinical metastases in the sentinel lymph nodes. It may be difficult to increase the number of cases in the short term. Patients will continue to be followed up and new cases will be included in time to increase the credibility of our data.

In conclusion, our study verified that the use of ICG surgical fluorescence imaging system for SLNB in patients with acral malignant melanoma is feasible and reliable, and the SLN detection rate of ICG is significantly higher than that of MB. The combined application of ICG and MB in medical institutions where radionuclides cannot be used may be an alternative solution.

\section{Acknowledgments}

Funding: None.

\section{Footnote}

Reporting Checklist: The authors have completed the STROBE reporting checklist. Available at https://dx.doi. org/10.21037/atm-21-4366

Data Sharing Statement: Available at https://dx.doi. org/10.21037/atm-21-4366

Conflicts of Interest: All authors have completed the ICMJE uniform disclosure form (available at https://dx.doi. org/10.21037/atm-21-4366). Dr. HC is an employee of the Nanjing Nuoyuan Medical Devices Co., Ltd., and provided the equipment of the ICG Surgical Fluorescent Imaging System. But the author promises that the paper was not affected during the writing process. The other authors have no conflicts of interest to declare.

Ethical Statement: The authors are accountable for all aspects of the work in ensuring that questions related to the accuracy or integrity of any part of the work are appropriately investigated and resolved. All the procedures were approved by local Ethics Committee of First Affiliated Hospital with Nanjing Medical University (2019-447). All participants signed the informed consent. The study was conducted in accordance with the Declaration of Helsinki (as revised in 2013).

Open Access Statement: This is an Open Access article distributed in accordance with the Creative Commons Attribution-NonCommercial-NoDerivs 4.0 International License (CC BY-NC-ND 4.0), which permits the noncommercial replication and distribution of the article with the strict proviso that no changes or edits are made and the original work is properly cited (including links to both the formal publication through the relevant DOI and the license). See: https://creativecommons.org/licenses/by-nc-nd/4.0/.

\section{References}

1. Guo J, Qin S, Liang J, et al. Chinese Guidelines on the Diagnosis and Treatment of Melanoma (2015 Edition). Ann Transl Med 2015;3:322.

2. Balch CM, Buzaid AC, Soong SJ, et al. Final version of the American Joint Committee on Cancer staging system for cutaneous melanoma. J Clin Oncol 2001;19:3635-48.

3. Morton DL, Wen DR, Wong JH, et al. Technical details of intraoperative lymphatic mapping for early stage melanoma. Arch Surg 1992;127:392-9.

4. Durham AB, Wong SL. Sentinel lymph node biopsy in melanoma: controversies and current guidelines. Future Oncol 2014;10:429-42.

5. Morton DL. Overview and update of the phase III Multicenter Selective Lymphadenectomy Trials (MSLT-I and MSLT-II) in melanoma. Clin Exp Metastasis 2012;29:699-706.

6. Morton DL, Wen DR, Foshag LJ, et al. Intraoperative lymphatic mapping and selective cervical lymphadenectomy for early-stage melanomas of the head and neck. J Clin Oncol 1993;11:1751-6.

7. Cousins A, Thompson SK, Wedding AB, et al. Clinical relevance of novel imaging technologies for sentinel lymph node identification and staging. Biotechnol Adv 2014;32:269-79.

8. Hettiaratchy S, Dheansa B, Powell B. Lymphatic mapping and sentinel lymph node biopsy in patients with melanoma of the lower extremity. Plast Reconstr Surg 2000;106:734-5.

9. Gershenwald JE, Scolyer RA, Hess KR, et al. Melanoma staging: Evidence-based changes in the American Joint Committee on Cancer eighth edition cancer staging manual. CA Cancer J Clin 2017;67:472-92. 
10. Stratmann SL, McCarty TM, Kuhn JA. Radiation safety with breast sentinel node biopsy. Am J Surg 1999;178:454-7.

11. Gil-Moreno A, Perez-Benavente A. Images in clinical medicine. Anaphylaxis associated with blue dye. N Engl J Med 2012;367:2026.

12. Niebling MG, Pleijhuis RG, Bastiaannet E, et al. A systematic review and meta-analyses of sentinel lymph node identification in breast cancer and melanoma, a plea for tracer mapping. Eur J Surg Oncol 2016;42:466-73.

13. Wang C, Tong F, Cao Y, et al. Long-term follow-up results of fluorescence and blue dye guided sentinel lymph node biopsy in early breast cancer. Breast Cancer Res Treat 2021;188:361-8.

14. Maramai M, Achilarre MT, Aloisi A, et al. Cervical reinjection of indocyanine green to improve sentinel lymph node detection in endometrial cancer. Gynecol Oncol 2021;162:38-42.

15. Shoji Y, Kumagai K, Kamiya S, et al. Prospective feasibility study for single-tracer sentinel node mapping by ICG (indocyanine green) fluorescence and OSNA (one-step nucleic acid amplification) assay in laparoscopic gastric cancer surgery. Gastric Cancer 2019;22:873-80.

16. Lafreniere AS, Shine JJ, Nicholas CR, et al. The use of indocyanine green and near-infrared fluorescence imaging to assist sentinel lymph node biopsy in cutaneous melanoma: A systematic review. Eur J Surg Oncol 2021;47:935-41.

17. Soft Tissue Tumor and Melanoma Group of Sarcoma Committee, Chinese Anti-Cancer Association. Chinese expert consensus on the surgical treatment of cutaneous/ acral melanoma V1.0. Zhonghua Zhong Liu Za Zhi 2020;42:81-93.

18. Chi Z, Li S, Sheng X, et al. Clinical presentation, histology, and prognoses of malignant melanoma in ethnic Chinese: a study of 522 consecutive cases. BMC Cancer 2011;11:85.

19. Leong SP, Morita ET, Südmeyer M, et al. Heterogeneous patterns of lymphatic drainage to sentinel lymph nodes by primary melanoma from different anatomic sites. Clin Nucl Med 2005;30:150-8.

20. Caracò C, Marone U, Celentano E, et al. Impact of false-negative sentinel lymph node biopsy on survival in patients with cutaneous melanoma. Ann Surg Oncol 2007;14:2662-7.

21. Carlson GW, Page AJ, Cohen C, et al. Regional recurrence after negative sentinel lymph node biopsy for melanoma. Ann Surg 2008;248:378-86.

22. Stoffels I, Dissemond J, Pöppel T, et al. Intraoperative
Fluorescence Imaging for Sentinel Lymph Node Detection: Prospective Clinical Trial to Compare the Usefulness of Indocyanine Green vs Technetium Tc 99m for Identification of Sentinel Lymph Nodes. JAMA Surg 2015;150:617-23.

23. Lam TK, Uren RF, Scolyer RA, et al. False-negative sentinel node biopsy because of obstruction of lymphatics by metastatic melanoma: the value of ultrasound in conjunction with preoperative lymphoscintigraphy. Melanoma Res 2009;19:94-9.

24. Gadd MA, Cosimi AB, Yu J, et al. Outcome of patients with melanoma and histologically negative sentinel lymph nodes. Arch Surg 1999;134:381-7.

25. Joseph E, Brobeil A, Glass F, et al. Results of complete lymph node dissection in 83 melanoma patients with positive sentinel nodes. Ann Surg Oncol 1998;5:119-25.

26. Wagner JD, Davidson D, Coleman JJ 3rd, et al. Lymph node tumor volumes in patients undergoing sentinel lymph node biopsy for cutaneous melanoma. Ann Surg Oncol 1999;6:398-404.

27. Scoggins CR, Chagpar AB, Martin RC, et al. Should sentinel lymph-node biopsy be used routinely for staging melanoma and breast cancers? Nat Clin Pract Oncol 2005;2:448-55.

28. Rychlik A, Zalewski K. Tracers and corresponding detection devices: technetium colloids, blue dyes \& NIR fluorescence. Chin Clin Oncol 2021;10:16.

29. Fujiwara M, Mizukami T, Suzuki A, et al. Sentinel lymph node detection in skin cancer patients using realtime fluorescence navigation with indocyanine green: preliminary experience. J Plast Reconstr Aesthet Surg 2009;62:e373-8.

30. Vahabzadeh-Hagh AM, Blackwell KE, Abemayor E, et al. Sentinel lymph node biopsy in cutaneous melanoma of the head and neck using the indocyanine green SPY Elite system. Am J Otolaryngol 2018;39:485-8.

31. Knackstedt R, Couto RA, Ko J, et al. Indocyanine Green Fluorescence Imaging with Lymphoscintigraphy for Sentinel Node Biopsy in Melanoma: Increasing the Sentinel Lymph Node-Positive Rate. Ann Surg Oncol 2019;26:3550-60.

32. Cloyd JM, Wapnir IL, Read BM, et al. Indocyanine green and fluorescence lymphangiography for sentinel lymph node identification in cutaneous melanoma. J Surg Oncol 2014;110:888-92.

33. Namikawa K, Yamazaki N. Sentinel lymph node biopsy guided by indocyanine green fluorescence for cutaneous melanoma. Eur J Dermatol 2011;21:184-90. 
34. Göppner D, Nekwasil S, Jellestad A, et al. Indocyanine green-assisted sentinel lymph node biopsy in melanoma using the "FOVIS" system. J Dtsch Dermatol Ges 2017;15:169-78.

35. Perone JA, Farrow N, Tyler DS, et al. Contemporary Approaches to In-Transit Melanoma. J Oncol Pract
2018;14:292-300.

36. Wolf IH, Richtig E, Kopera D, et al. Locoregional cutaneous metastases of malignant melanoma and their management. Dermatol Surg 2004;30:244-7.

(English Language Editor: A. Kassem)

Cite this article as: Jiang K, Luo B, Hou Z, Li C, Cai H, Tang J, Yao G. Application of an indocyanine green surgical fluorescence imaging system in sentinel lymph node biopsy of acral malignant melanoma. Ann Transl Med 2021;9(18):1456. doi: 10.21037/atm-21-4366 